\title{
Resistance to antimicrobials and vancomycin treatment in neonatal sepsis in a hospital in Brazil
}

\author{
Rafael Hoffmann SILVEIRA ${ }^{1}$, Kelly Goulart LIMA ${ }^{2}$ (D), \\ Carolina Heloisa dos SANTOS-BOROWICZ ${ }^{2}$, Karin Hepp SCHWAMBACH ${ }^{2}$ (D), Hugo BOCK ${ }^{1}$ \\ ${ }^{1}$ Universidade do Vale do Rio dos Sinos, São Leopoldo/RS; ${ }^{2}$ Hospital Materno Infantil Presidente Vargas \\ Corresponding author: Lima KG, keglima@gmail.com
}

Submitted: 19-02-2020 Resubmitted: 10-08-2020 Accepted:10-08-2020

Peer review: blind reviewer and Maria Garbi Novaes

\begin{abstract}
Objective: To describe the antimicrobial resistance profile of strains isolated from neonates with septicemia undergoing vancomycin treatment, to determine the agreement of treatment with guideline and blood cultures, to assess the presence of acute kidney injury (AKI) and the clinical outcome in a hospital in Brazil. Methods: A total of 51 newborns treated with vancomycin were studied in the period from January to December 2017. Treatment assessment was verified by the compliance with the Rational Use and Insurance Policy of Antibiotics in Neonatology and blood culture. The presence of AKI was based on the results of serum creatinine. The data were monitored by the computerized system of the laboratory. Results: The main isolated strain was Staphylococcus (coagulase negative) (82.9\%), 93.5\% with resistance to oxacillin for early infections and $100 \%$ for late infections. The same strain showed $54.8 \%$ resistance to gentamicin for cases of early sepsis and $66.6 \%$ for late sepsis. There were no cases of resistance to vancomycin. The treatments agreed with the reference protocol in $46.3 \%$ and with the results of blood cultures in $56 \%$. Only two cases of AKI are observed. Conclusions: The oxacillin-resistant coagulase-negative staphylococcus strains were the main causes of neonatal sepsis. The use of gentamicin for sensitive strains may reduce the use of vancomycin. The results suggest the need for better standardization of the treatment to reduce the risk of toxicity and rational use of therapeutic resources.
\end{abstract}

Keywords: neonatal sepsis, vancomycin, antimicrobial drug resistance, blood culture.

\section{Resistência a antimicrobianos e tratamento com vancomicina na sepse neonatal em hospital do Brasil}

\begin{abstract}
Resumo
Objetivos: Avaliar o perfil de resistência aos antimicrobianos das cepas isoladas de neonatos com septicemia em tratamento com vancomicina, determinar a concordância do tratamento com o protocolo referência e com as hemoculturas, avaliar a presença de lesão renal aguda (LRA) e o desfecho clínico em um hospital no Brasil. Métodos: Estudou-se 51 neonatos submetidos ao tratamento com vancomicina no período de janeiro a dezembro de 2017. A avaliação do tratamento foi baseada na verificação da sua concordância com o Protocolo de Uso Racional e Seguro de Antibióticos em Neonatologia e com a hemocultura. A determinação da presença de LRA foi baseada nos resultados de creatinina sérica. Os dados foram obtidos do prontuário e do sistema informatizado do laboratório. Resultados: A principal cepa isolada foi Staphylococcus (coagulase negativa) (82,9\%), a qual apresentou 93,5\% de resistência à oxacilina para as infecções precoces e $100 \%$ para as infecções tardias. A mesma cepa apresentou 54,8\% de resistência à gentamicina para os casos de sepse precoce e $66,6 \%$ para sepse tardia. Não houveram casos de resistência à vancomicina. Os tratamentos concordaram com o protocolo referência em $46,3 \%$ e com os resultados das hemoculturas em $56 \%$. Foram observados apenas dois casos de LRA. Conclusões: As cepas de estafilococos coagulase negativa resistentes à oxacilina foram as principais causadoras da sepse neonatal. O uso de gentamicina para as cepas sensíveis pode reduzir o uso de vancomicina. Os resultados sugerem a necessidade de maior padronização do tratamento para redução do risco de toxicidade e uso racional dos recursos terapêuticos.
\end{abstract}

Palavras-chave: sepse neonatal, vancomicina, resistência microbiana a medicamentos, hemocultura. 


\section{Introduction}

Antimicrobial agents correspond to a class of drugs widely used in hospitals, accounting for $20 \%-50 \%$ of drug expenditures. At the same time, they are the only pharmacological agents that not only affect patients who use them, but also significantly interfere in the hospital setting due to changes in microbial ecology. ${ }^{1}$ The association between the inappropriate use of antimicrobial agents and the increased occurrence of infections associated with the hospital setting caused by resistant germs in neonatal units has already been demonstrated. ${ }^{2}$

Vancomycin is an antibiotic for hospital use, of the tricyclic glycopeptide class, whose mechanism of action is the inhibition of cell wall synthesis of Gram-positive bacteria. This drug is indicated for the treatment of infections caused by the methicillin-resistant Staphylococcus aureus (MRSA) bacteria, systemic staphylococcal infections in patients allergic to $\beta$-lactams, infections caused by enterococci and pneumococci resistant to penicillin and as an alternative for the treatment of enterocolitis by Clostridium difficile. ${ }^{3}$ In neonatology, this drug is widely prescribed for the treatment of late neonatal sepsis or late bloodstream infections, empirically or when Gram-positive bacteria are isolated in blood cultures. ${ }^{4}$

Neonatal sepsis is characterized by clinical signs of infection in the first month of life, accompanied by bacteremia, with or without positive blood culture. Early neonatal sepsis occurs in the first 48 hours of life; and late, after that period. The etiology of early neonatal sepsis is usually associated with vertical contamination. On the other hand, late neonatal sepsis can be caused by microorganisms in the maternal genital tract or in the hospital setting, or by other environmental risk factors. ${ }^{5}$

In case of suspected neonatal sepsis, complementary tests such as blood culture, blood count, C-Reactive Protein (CRP), urinalysis, urine culture, lumbar puncture, and chest $x$-ray can help confirm the diagnosis. Blood culture is the test requested to determine bacteremia and must be requested before the start of antibiotic therapy to avoid false negatives. ${ }^{6}$

Approximately $50 \%$ of the medical prescriptions for antimicrobials are improperly done. The excessive use of these drugs is not only associated with the emergence and selection of resistant bacteria, but also with adverse events, as well as with increased costs, morbidity and mortality. ${ }^{2}$ With the emergence of resistant enterococcal strains and intermediate-resistant Staphylococcus aureus, studies on the use of antimicrobials can contribute to increasing attention on the use of these drugs, as well as to expanding discussions about the potential risks that inadequate prescription may cause. ${ }^{7}$

The objectives of this study were to evaluate the antimicrobial resistance profile of strains isolated from neonates with septicemia undergoing vancomycin treatment in a maternal-child hospital in Porto Alegre, RS, Brazil, and the treatment agreement with the reference protocol and the results blood cultures.

\section{Methods}

This is a retrospective observational study carried out in a maternalchild hospital in southern Brazil. The sample was selected for convenience, with an analysis of 503 medical records of neonates admitted to the neonatal ICU in the period from January $1^{\text {st }}, 2017$ to December 31 ${ }^{\text {st }}$, 2017. All the neonatal ICU patients who used vancomycin during hospitalization and underwent blood culture during the study period were included, totaling 51 patients.

The data were collected in an institutional database, including medical records and results of laboratory tests. The analyzed variables were sepsis classification, bacterial identification, antimicrobial sensitivity profile, treatment protocol, and case outcomes. In this study, criteria for the diagnosis of sepsis were adopted according to the date of the first blood culture collection or the start of antibiotic therapy, when the second precedes the first. The classification of sepsis and the treatment protocol followed the description of the SBP. ${ }^{8}$

Bacterial sensitivity to antimicrobial agents was determined by the hospital's laboratory using the automated broth microdilution method and identification by the automated colorimetry method. Sensitivity tests to antimicrobials performed in blood culture follow the standards described by the CLSI (The Clinical \& Laboratory Standards Institute). The assessment of the presence of acute kidney injury was based on the KDIGO (Kidney Disease Improving Global Outcomes) classification, in which serial plasma creatinine is used within a period of 7 days to determine the stage of kidney injury. ${ }^{9}$

The data were stored in a database created in the Microsoft Office Excel 2013 software and the statistical analysis was performed using the IBM SPSS Statistics 20 program. The data were submitted to simple descriptive analysis and are presented in tables of absolute and relative frequency. The relationships between qualitative variables were analyzed using Pearson's or Fisher's Chi-square test, with $\mathrm{p}<0.05$ being considered as a statistically significant difference.

The research was approved by the Research Ethics Committee of the Presidente Vargas Maternal-Child Hospital under number 2.965 .683$.

\section{Results}

Data from 51 patients who used vancomycin and underwent blood culture during their neonatal ICU stay were analyzed. Of these, $16(31.4 \%)$ presented negative results in all the blood cultures performed. In 35 cases (68.6\%), there was positivity for some type of microorganism. The profile of the cases studied in relation to the sensitivity tests is described in Figure 1.

The frequency of agreement of the therapy with the SBP protocol was $46.3 \%(n=19)$ and with the blood culture results, 56\% $(n=23)$. The reasons for not agreeing with the protocol were the following: sensitivity to another indicated antibiotic (47.6\%), use of vancomycin as initial therapy (28.6\%), use of vancomycin as initial therapy and sensitivity to another indicated antibiotic (23.8\%). The reason for not agreeing with the results of the blood cultures was due to sensitivity to other antibiotics. Of the total number of patients studied, empirical treatment with gentamicin and ampicillin was initiated in $4(7.8 \%)$ before the blood culture was collected. In 14 patients (27.5\%), treatment was started at least one day after the first blood culture collection and, in 33 patients $(64.7 \%)$, therapy was started on the same date as the first collection.

Considering the total number of patients studied ( $n=51$ ), the type of sepsis was classified as early for 42 patients $(82.4 \%$ of 
Figure 1. Profile of the blood culture results.

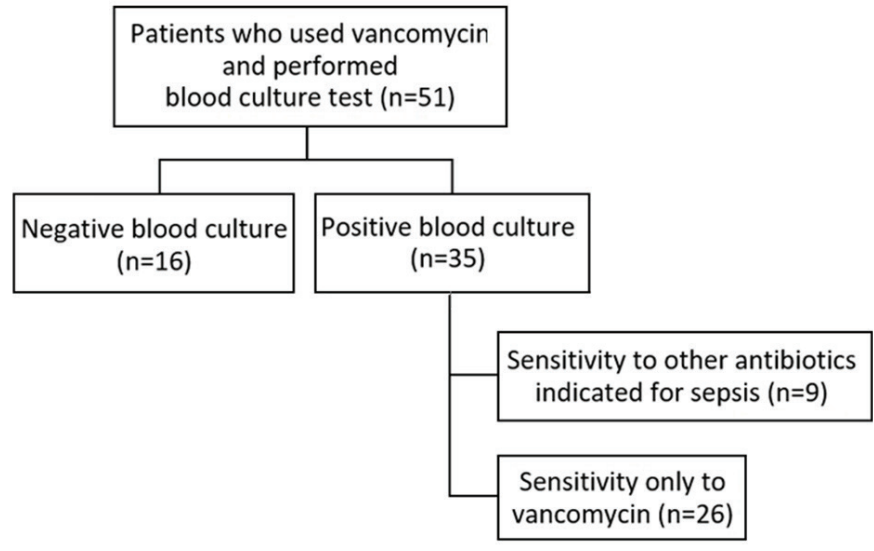

the total). Of these, 28 (66.7\%) had positive blood culture. Nine patients (17.6\% of the total) were classified as late sepsis and, of these, $6(66.7 \%)$ presented positive results in blood culture. Of the total patients included in the study, 6 had more than one positive blood culture. Considering all the positive blood cultures $(n=41)$, the sensitivity profile of the microorganisms identified in the blood cultures by type of sepsis is described in Table 1.

Of the 41 positive blood cultures, 35 were from patients with early sepsis, and 6 from late sepsis. All the isolated Gram-positive microorganisms showed sensitivity to vancomycin. The main isolated strain belongs to the Staphylococcus Group (coagulase negative) $(82.9 \%, n=34)$, which presented $93.5 \%$ resistance to oxacillin for early infections and $100 \%$ for late infections. The same strain showed $54.8 \%$ resistance to gentamicin for cases of early sepsis and $66.6 \%$ for late sepsis, as shown in Table 1.

Table 1. Sensitivity profile of the microorganisms identified in blood cultures by type of sepsis ( $n=41)$.

\begin{tabular}{|c|c|c|c|c|c|c|c|c|c|c|}
\hline \multirow{2}{*}{ Microorganisms by type of sepsis } & \multirow{2}{*}{$\begin{array}{l}\text { All } \\
\%(n)\end{array}$} & \multicolumn{3}{|c|}{$\begin{array}{c}\text { Oxacillin/Ampicillin } \\
\% \text { (n) }\end{array}$} & \multicolumn{3}{|c|}{$\begin{array}{l}\text { Gentamycin } \\
\%(n)\end{array}$} & \multicolumn{3}{|c|}{$\begin{array}{l}\text { Amikacin } \\
\%(n)\end{array}$} \\
\hline & & $\mathbf{R}$ & $S$ & I & $\mathbf{R}$ & $S$ & I & $\mathbf{R}$ & $S$ & 1 \\
\hline Early sepsis & $85.4(35)$ & $94.1(32)$ & $5.9(2)$ & - & $57.1(20)$ & $34.3(12)$ & $8.6(3)$ & $75.0(3)$ & $25.0(1)$ & - \\
\hline CNS & $88.6(31)$ & $93.5(29)$ & $6.5(2)$ & - & $54.8(17)$ & $35.5(11)$ & $9.7(3)$ & NA & NA & NA \\
\hline Staphylococcus epidermidis & $42.9(15)$ & $93.3(14)$ & $6.7(1)$ & - & $53.3(8)$ & $26.7(4)$ & $20.0(3)$ & NA & NA & NA \\
\hline Staphylococcus capitis & $14.3(5)$ & $100.0(5)$ & - & - & $100.0(5)$ & - & - & NA & NA & NA \\
\hline SND & $14.3(5)$ & $80.0(4)$ & $20.0(1)$ & - & $60.0(3)$ & $40.0(2)$ & - & NA & NA & NA \\
\hline Staphylococcus hominis & $11.4(4)$ & $100.0(4)$ & - & - & - & $100.0(4)$ & - & NA & NA & NA \\
\hline Staphylococcus haemolyticus & $2.9(1)$ & $100.0(1)$ & - & - & $100.0(1)$ & - & - & NA & NA & NA \\
\hline Staphylococcus hom. + SE & $2.9(1)$ & $100.0(1)$ & - & - & - & $100.0(1)$ & - & NA & NA & NA \\
\hline Enterobacter cloacae c. & $5.7(2)$ & $100.0(2)$ & - & - & $50.0(1)$ & $50.0(1)$ & - & $50.0(1)$ & $50.0(1)$ & - \\
\hline Acinetobacter baumannii c. & $2.9(1)$ & NA & NA & NA & $100.0(1)$ & - & - & $100.0(1)$ & - & - \\
\hline Klebsiella oxytoca ESBL + & $2.9(1)$ & $100.0(1)$ & - & - & $100.0(1)$ & - & - & $100.0(1)$ & - & - \\
\hline Late sepsis & $14.6(6)$ & $100.0(5)$ & - & - & $80.0(4)$ & - & $20.0(1)$ & $100.0(2)$ & - & - \\
\hline Staphylococcus epidermidis & $50.0(3)$ & $100.0(3)$ & - & - & $66.6(2)$ & - & $33.3(1)$ & NA & NA & NA \\
\hline Acinetobacter baumannii c. $+S E$ & $16.7(1)$ & $100.0(1)$ & - & - & $100.0(1)$ & - & - & $100.0(1)$ & - & - \\
\hline Serratia marcescens & $16.7(1)$ & $100.0(1)$ & - & - & $100.0(1)$ & - & - & $100.0(1)$ & - & - \\
\hline Streptococcus viridans ${ }^{a}$ & $16.7(1)$ & NA & NA & NA & NA & NA & NA & NA & NA & NA \\
\hline
\end{tabular}

NOTE: SND: Species not defined. CNS: Staphylococcus group (coagulase negative). Acinetobacter baumannii c.: Acinetobacter baumannii complex. Acinetobacter baumannii c + SE: Acinetobacter baumannii complex + Staphylococcus epidermidis. Staphylococcus hom. + SE: S. hominis + S. epidermidis. Enterobacter cloacae c.: Enterobacter cloacae complex. ESBL: Extended Spectrum Beta-Lactamase. R: Resistant, S: Sensitive, I: Intermediate, NA: Not Applicable. aPresented sensitivity only to vancomycin and resistance to cefotaxime, ceftriaxone and cefepime. Presented intermediate sensitivity to penicillin. Performed more than one blood culture: 5 patients with early sepsis and one patient with late sepsis.

In early sepsis, we observed a case of sensitivity and a case of resistance to the cefotaxime antimicrobial agent for the Enterobacter cloacae complex strain and a case of resistance for Acinetobacter baumannii complex. The results of the sensitivity assessment against cefepime showed that three strains showed resistance (Acinetobacter baumannii complex, Klebsiella oxytoca ESBL+, and Enterobacter cloacae complex), and a strain of Enterobacter cloacae complex showed sensitivity. In cases of late sepsis, the same result obtained with cefotaxime was observed.

In cases of late sepsis, the Serratia marcescens, Streptococcus viridans, and Acinetobacter baumannii complex microorganisms were resistant to cefotaxime in all the samples. The same result was obtained for cefepime. All the patients, including early and late sepsis, used vancomycin associated with other antimicrobial agents, the association with amikacin being the most frequent. The vancomycin treatment period ranged from 1 to 37 days, with the majority $(73.2 \%)$ receiving treatment for more than 10 days.
The other prescriptions included gentamicin, ampicillin, cefepime, meropenem, ampicillin associated with sulbactam, and penicillin.

Considering only patients who had positive blood cultures $(n=41)$, the main initial empirical therapy chosen was the combination of gentamicin and ampicillin (treatment $1, n=28,68.3 \%$ ). The other treatments were penicillin and gentamicin (treatment 2, n=2, $4.9 \%$ ), and vancomycin (treatment $3, n=11,26.8 \%$ ). The sensitivity profile of the isolated microorganisms in view of the empirical therapies and outcome by type of sepsis is shown in Table 2.

When evaluating all patients in the study, even those with negative blood cultures, of the 17 cases that started therapy with vancomycin, 5 preceded the first positive blood culture ( 3 cases of early sepsis and 2 of late sepsis), and 5 did not present positive blood cultures ( 3 cases of early sepsis and 2 of late sepsis). The outcomes verified for the studied cases were $80.3 \%$ of hospital discharge (41 cases) and only 4 patients progressed to death. 
Table 2. Sensitivity profile of the isolated microorganisms in view of empirical therapies and outcome by type of sepsis ( $\mathrm{n}=41$ ).

\begin{tabular}{|c|c|c|c|c|c|c|c|}
\hline \multirow{2}{*}{$\begin{array}{l}\text { Microorganism by type of sepsis and sensitivity } \\
\text { profile }\end{array}$} & \multirow{2}{*}{$\begin{array}{l}\text { All } \\
\%(n)\end{array}$} & \multirow{2}{*}{$\frac{\mathrm{T1}}{\%(\mathrm{n})}$} & \multirow{2}{*}{$\begin{array}{l}\mathrm{T2} \\
\%(\mathrm{n})\end{array}$} & \multirow{2}{*}{$\begin{array}{l}\text { T3 } \\
\%(n)\end{array}$} & \multicolumn{3}{|c|}{ Outcome \% (n) } \\
\hline & & & & & Discharge & Death & NI \\
\hline Early sepsis & $85.4(35)$ & $71.4(25)$ & $5.7(2)$ & $22.9(8)$ & $77.1(27)$ & $8.6(3)$ & $14.3(5)$ \\
\hline \multicolumn{8}{|c|}{ Staphylococcus Group (coagulase negative) - CNS } \\
\hline Staphylococcus epidermidis & 42.9 (15) & $66.7(10)$ & $6.6(1)$ & $26.7(4)$ & $80.0(12)$ & $13.3(2)$ & $6.7(1)$ \\
\hline Sensitive & $40.0(6)$ & $20.0(2)$ & - & $100.0(4)$ & $33.3(4)$ & $50.0(1)$ & $100.0(1)$ \\
\hline Resistant & $46.6(7)$ & $60.0(6)$ & $100.0(1)$ & - & $50.0(6)$ & $50.0(1)$ & - \\
\hline Intermediate & $13.4(2)$ & $20.0(2)$ & - & - & $16.7(2)$ & - & - \\
\hline Staphylococcus capitis & $14.3(5)$ & $100.0(5)$ & - & - & $80.0(4)$ & - & $20.0(1)$ \\
\hline Resistant & $100.0(5)$ & $100.0(5)$ & - & - & $100.0(4)$ & - & $100.0(1)$ \\
\hline SND & $14.3(5)$ & $40.0(2)$ & - & $60.0(3)$ & $60.0(3)$ & $20.0(1)$ & $20.0(1)$ \\
\hline Sensitive & $80.0(4)$ & $50.0(1)$ & - & $100.0(3)$ & $66.7(2)$ & $100.0(1)$ & $100.0(1)$ \\
\hline Resistant & $20.0(1)$ & $50.0(1)$ & - & - & $33.3(1)$ & - & - \\
\hline Staphylococcus hominis & $11.4(4)$ & $100.0(4)$ & - & - & $50.0(2)$ & - & $50.0(2)$ \\
\hline Sensitive & $100.0(4)$ & $100.0(4)$ & - & - & $100.0(2)$ & - & $100.0(2)$ \\
\hline Staphylococcus haem. & $2.9(1)$ & $100.0(1)$ & - & - & $100.0(1)$ & - & - \\
\hline Resistant & $100.0(1)$ & $100.0(1)$ & - & - & $100.0(1)$ & - & - \\
\hline Staphylococcus hom. + SE & $2.9(1)$ & $100.0(1)$ & - & - & $100.0(1)$ & - & - \\
\hline Sensitive & $100.0(1)$ & $100.0(1)$ & - & - & $100.0(1)$ & - & - \\
\hline Enterobacter cloacae complex & $5.7(2)$ & - & $50.0(1)$ & $50.0(1)^{*}$ & $100.0(2)^{*}$ & - & - \\
\hline Resistant & $100.0(1)$ & - & $100.0(1)$ & - & $100.0(1)$ & - & - \\
\hline Acinetobacter baumannii c. & $2.9(1)$ & $100.0(1)$ & - & - & $100.0(1)$ & - & - \\
\hline Resistant & $100.0(1)$ & $100.0(1)$ & - & - & $100.0(1)$ & - & - \\
\hline Klebsiella oxytoca ESBL+ & $2.9(1)$ & $100.0(1)$ & - & - & $100.0(1)$ & - & - \\
\hline Resistant & $100.0(1)$ & $100.0(1)$ & - & - & $100.0(1)$ & - & - \\
\hline Late sepsis & $14.6(6)$ & $50.0(3)$ & - & $50.0(3)$ & $66.6(4)$ & - & $33.4(2)$ \\
\hline Staphylococcus epidermidis & $50.0(3)$ & $33.3(1)$ & - & $66.7(2)$ & $100.0(3)$ & - & - \\
\hline Sensitive & $66.7(2)$ & - & - & $100.0(2)$ & $66.7(2)$ & - & - \\
\hline Resistant & $33.3(1)$ & $100.0(1)$ & - & - & $33.3(1)$ & - & - \\
\hline Acinetobacter baumannii c. $+S E$ & $16.7(1)$ & $100.0(1)$ & - & - & $100.0(1)$ & - & - \\
\hline Resistant & $100.0(1)$ & $100.0(1)$ & - & - & $100.0(1)$ & - & - \\
\hline Serratia marcescens & $16.7(1)$ & - & - & $100.0(1)^{*}$ & - & - & $100.0(1)^{*}$ \\
\hline Streptococcus viridans & $16.7(1)$ & $100.0(1)$ & - & - & - & - & $100.0(1)$ \\
\hline Intermediate & $100.0(1)$ & $100.0(1)$ & - & - & - & - & $100.0(1)$ \\
\hline
\end{tabular}

NOTE: SND: Species not defined. CNS: Staphylococcus group (coagulase negative). Acinetobacter baumannii c.: Acinetobacter baumannii complex. Acinetobacter baumannii c + SE: Acinetobacter baumannii complex + Staphylococcus epidermidis. Staphylococcus hom. + SE: Staphylococcus hominis + Staphylococcus epidermidis. Staphylococcus haem.: Staphylococcus haemolyticus. ESBL: Extended Spectrum Beta-Lactamase. T1: Gentamicin and Ampicillin Association. T2: Gentamicin and Penicillin Association. T3: Vancomycin. NI: Not Informed in the medical record. *Initial treatment with vancomycin, but this antimicrobial agent was not tested, since the isolated bacterium was a gram-negative bacillus. NI: Not Informed in the medical record. In the sensitivity information, lines where there was no result were excluded.

The assessment of renal function was performed in $58.8 \%$ of the patients using the KDIGO classification based on the measurement of serum creatinine. Of these, $33.3 \%$ had only one result of serum creatinine, not allowing for the classification of possible acute kidney injury. Acute kidney injury was classified as zero stage in $60 \%$ of the cases. Only 1 patient was classified as stage 1 (increase in creatinine greater than $0.3 \mathrm{mg} / \mathrm{dL}$ ) and another as stage 3 (increase greater than 200\% of the previous result).

The statistical analysis of the relationship between the classification of the type of sepsis and the outcome did not show any significant difference between the early and late sepsis groups $(p=0.572)$. There was also no relationship between agreement with the treatment protocol and the outcome ( $p=0.555)$; as well as between the agreement of treatment with blood culture and the outcome $(p=0.112)$. The data are presented in Table 3.
Table 3. Relationship between sepsis classification, treatment protocol, blood cultures, and outcomes $(n=41)$.

\begin{tabular}{|c|c|c|c|c|c|}
\hline \multirow{2}{*}{$\begin{array}{l}\text { Evaluated } \\
\text { variables }\end{array}$} & \multirow[t]{2}{*}{$\%(n)$} & \multicolumn{3}{|c|}{ Outcome \% (n) } & \multirow[t]{2}{*}{ p-value } \\
\hline & & Discharge & Death & NI & \\
\hline All & $100.0(41)$ & $75.6(31)$ & $7.3(3)$ & $17.0(7)$ & \\
\hline \multicolumn{5}{|c|}{ Sepsis classification } & 0.572 \\
\hline Early & $85.4(35)$ & $77.1(27)$ & $8.6(3)$ & $14.3(5)$ & \\
\hline Late & $14.6(6)$ & $66.6(4)$ & - & $33.4(2)$ & \\
\hline \multicolumn{5}{|c|}{ Agreement with treatment protocol } & 0.555 \\
\hline Yes & $46.3(19)$ & $51.6(16)$ & $33.3(1)$ & $28.6(2)$ & \\
\hline No & $53.7(22)$ & $48.4(15)$ & $66.7(2)$ & $71.4(5)$ & \\
\hline \multicolumn{5}{|c|}{ Agreement of treatment with blood culture } & 0.112 \\
\hline Yes & $56.0(23)$ & $58.0(18)$ & $100.0(3)$ & $28.6(2)$ & \\
\hline No & $44.0(18)$ & $42.0(13)$ & - & $71.4(5)$ & \\
\hline
\end{tabular}

NOTE: NI: Not Informed in the medical record. *Some patients performed more than one blood culture. 


\section{Discussion}

Most of the patients who used vancomycin to treat neonatal sepsis tested positive for blood culture. The cases in which the treatment was continued even with a negative blood culture result are justified due to limitations in the sensitivity of the examination. In this context, the volume of blood collected is one of the most critical variables for positivity, since low volumes can reduce sensitivity. On the other hand, the lower the newborn's weight, the lower the volume recommended for collection. ${ }^{10}$ Since the estimated blood culture sensitivity is $80 \%$, when the patient has symptoms and there is no clinical improvement, the full course of the antibiotic is recommended, regardless of the result of blood culture. ${ }^{6}$ Another factor that can interfere with the result of blood cultures is the beginning of antibiotic therapy before collection, which occurred in rare cases. According to the Pan American Health Organization, blood cultures should be collected before the antibiotic is used to avoid this interference in the diagnosis. ${ }^{11}$

Considering the cases with positive blood cultures, approximately half of the treatments performed agreed with the recommendations of therapy for neonatology (46.3\%). ${ }^{6}$ The discordant cases were those in which the treatment with vancomycin was carried out, despite the microorganism being sensitive to another antibiotic or showing no indication for the use of this drug, as in the treatment of sepsis caused by enterobacteria. The discordant cases were the following: sensitive to gentamicin (10 cases- $71.4 \%$ ), sensitive to gentamicin and oxacillin ( 2 cases - 14.3\%), and enterobacteria with no indication for vancomycin use (2 cases - 14.3\%). Patients with sepsis caused by enterobacteria had empirical therapy with vancomycin subsequently discontinued and the appropriate treatment was prescribed, but a more rigorous assessment can prevent this exposure and possible cases of drug resistance.

The profile of the microorganisms identified in blood cultures of neonates showed that the antibiogram could help reduce vancomycin use since, when identifying a microorganism sensitive to gentamicin or oxacillin, the indication is to suspend vancomycin use and administer any of these antimicrobial agents, which have less toxicity. ${ }^{12}$ On the other hand, most treatments with vancomycin are in accordance with the results of blood cultures (56\%), since most of the strains showed resistance to other antimicrobial agents and sensitivity only to vancomycin.

The empirical use of vancomycin can be associated with the prevalence of the coagulase negative Staphylococcus (CNS) group, which had a high rate of resistance to oxacillin and moderate resistance to gentamicin in the studied population. However several studies indicate the need for rational use of this drug. ${ }^{13-15}$ In a study conducted for 7 years in the Netherlands, the majority of the patients with sepsis caused by CNS were successfully treated with cefazolin and the use of vancomycin can be restricted to specific cases. Despite the use of cefazolin for a long period of time, the minimum inhibitory concentration values remained low and in the susceptible range. ${ }^{13}$ In the United States, the evaluation of a protocol for of vancomycin use in two hospitals demonstrated that the exposure of newborns to vancomycin was significantly reduced without affecting patient safety in the short term. ${ }^{14}$ The greatest use of antibiotics in the Neonatal ICU occurs as an empirical therapy. Therefore, it is important to evaluate strategies to reduce the general use of vancomycin from the beginning, and not just interrupt therapy, when the cultures do not generate a pathogen susceptible only to vancomycin. ${ }^{15}$
The CNS group was the most prevalent in the results of blood cultures. Our results are similar to studies conducted in Brazil and in the United States which show that the main microorganisms that cause late sepsis in premature babies in Neonatal ICUs belong to this group. ${ }^{16-18}$ The vast majority of these microorganisms isolated in our study showed resistance to $\beta$-lactams, making the use of vancomycin suitable for proven infections.

Although we did not find vancomycin-resistant microorganisms in our blood culture results, there are reports in the literature of emergency resistance to this drug by methicillin-resistant Staphylococcus aureus and Enterococcus faecium. One of these studies described an outbreak caused by vancomycin-resistant Enterococcus in a Neonatal ICU from Greece. ${ }^{19}$ Another study in France showed that a clonal population of $S$. capitis resistant to methicillin and with reduced sensitivity to vancomycin has spread in several neonatal ICUs. ${ }^{20}$ These studies reinforce the importance of reducing the empirical use of vancomycin to prevent the development of resistance. ${ }^{15}$

Most of the patients were classified as early sepsis, who predominantly presented the CNS microorganism in the blood cultures (88.6\%). Previous studies have found results different from ours, since they reported a predominance of Gram-negative pathogens as causing early sepsis. ${ }^{21,22}$ The main microorganisms involved in early sepsis described in the literature are group B Streptococcus and Escherichia coli, which are colonizers of the maternal genital tract and can contaminate the amniotic fluid, placenta or vaginal canal. On the other hand, coagulase-negative Staphylococcus are more often associated with late sepsis, which is related to possible nosocomial contamination in the neonatal period, especially in patients with very low birth weight. ${ }^{23}$ Thus, most of the studied patients with early sepsis may also have nosocomial contamination as the source of the infection.

Our results from patients with late sepsis were similar to other studies, since most of the isolated microorganisms (50\%) were identified as coagulase negative Staphylococcus ${ }^{16,18}$. One of those studies that evaluated 6,956 neonates with late sepsis seen at the National Institute of Child Health and Human Development Neonatal Research Network's clinical centers found that $48 \%$ of the infections were caused by $\mathrm{CNS}^{16}$. Another study that included 357 neonates with late-onset sepsis in Brazil also found a predominance of CNS as an etiological agent since, in $60 \%$ of the cases, microorganisms from this group were isolated. ${ }^{18}$ In addition, all of these isolates were resistant to beta-lactams, as mentioned in a previous study. ${ }^{15}$

Of the 17 cases that started vancomycin therapy, 5 preceded the first positive blood culture and 5 did not show positive blood cultures, suggesting the empirical use of vancomycin in disagreement with the results of laboratory tests. Regarding the period of treatment with vancomycin, it showed a high variation. Our records demonstrate that extremely short treatments are related to death and that long treatments were those that had small intervals during the intervention.

In most of the cases evaluated, treatment with vancomycin lasted more than 10 days (73.2\%). In a cohort study carried out with 126 very low birth weight neonates in a tertiary hospital in Israel, it was shown that the predominant treatment period was 5 days (38.1\%), followed by 6 to 7 days (25.4\%) and by 8 to 10 days (24.6\%). Treatments longer than 10 days were used by $11.9 \%$ of the patients, who were diagnosed with infective endocarditis or aortic thrombosis. Treatment of uncomplicated sepsis with 
vancomycin for 5 days after the last positive blood culture appears to be associated with a satisfactory result. ${ }^{24}$ Prolonged antibiotic therapy in high-risk pre-term infants is associated with changes in the microbiome and, in relation to CNS, the duration of therapy from three to five days can be sufficient. ${ }^{15}$

Most of the patients (58.8\%) underwent renal function assessment through the measurement of serum creatinine; however, efforts to expand the monitoring of this marker can improve care for these patients. In addition, the serial request for serum creatinine can be extended to enable classification, as described in the literature. ${ }^{9}$ Considering the patients evaluated, vancomycin use can be related to the development of acute kidney injury in rare cases, as only two patients were classified as stage greater than zero. A previous study with pediatric patients reported acute kidney injury related to vancomycin use in $12.2 \%$ of the patients, a higher figure than that found in our study; however, this difference can be related to the difference in the number of patients studied and to differences in the age group..$^{25}$

The low death rate of the sample showed high effectiveness in treating neonates with sepsis. Of the cases that progressed to death, 2 patients presented positive blood cultures for $S$. epidermidis, 1 for CNS, and there was 1 case with negative blood culture. They were treated with vancomycin 100\% in agreement with the antibiograms, since the isolated microorganisms were resistant to the other antibiotics.

This study had some limitations. Its development in a single hospital and with a small sample can decrease the external validity of the results. The collection of blood cultures after the beginning of antibiotic therapy that occurred in rare cases and the use of vancomycin as the first therapeutic choice can be considered confounding factors, hindering interpretation of the results. Regarding the technical limitation related to blood culture sensitivity, the association of quantitative real-time PCR (qPCR) could assist in the diagnosis. GPCR has high sensitivity and specificity, and would not be impaired by the use of antimicrobial agents before collection or by the viability of the microorganism at the time of collection. This tool has already been used in specialized centers for tuberculosis and could significantly assist in the diagnosis and monitoring of neonatal sepsis treatment. Despite having a higher cost, in the case of neonatology, the speed of identification could direct the correct therapy, which would reduce the length of hospital stay and eventually, would generate a lower cost. ${ }^{26}$

The statistical analysis showed no relationship between the outcome and the classification of the type of sepsis, nor with the treatment protocol, nor with blood cultures. This may have occurred due to the limited number of patients studied, requiring further studies. Our study has the prospect of expansion with the assessment of serum vancomycin concentration in neonate patients diagnosed with sepsis in order to relate the results of laboratory and clinical examinations.

\section{Conclusion}

The CNS strains resistant to oxacillin were the main causes of neonatal sepsis. Gentamicin has been shown to be a therapeutic option in some cases, and can reduce the empirical use of vancomycin. The results of this study can guide the planning of strategies to reduce the use of antibiotics with greater potential for toxicity such as vancomycin and, at the same time, assist in the choice and maintenance of the most appropriate therapy.

\section{Funding source}

The authors declare that the research did not receive funding for its realization.

\section{Collaborators}

RHS and KGL contributed in data collection, data analysis, and in the writing of the manuscript. CHSB contributed in data collection and in the review of the manuscript. KHS contributed in data analysis and in the review of the manuscript. $\mathrm{HB}$ contributed in data analysis, research coordination, and review of the manuscript.

\section{Conflict of interests statement}

The authors declare that there is no conflict of interest in relation to this article.

\section{References}

1. Rodrigues FD, Bertoldi AD. Perfil da utilização de antimicrobianos em um hospital privado. Cien Saude Colet. 2010;15(suppl 1):1239-47.

2. Silva ACB, Anchieta LM, Lopes MFD, et al. Inadequate use of antibiotics and increase in neonatal sepsis caused by resistant bacteria related to health care assistance: a systematic review. Brazilian J Infect Dis.2018;22(4):328-37.

3. Tavares W. Manual de antibióticos e quimioterápicos antiinfecciosos. 2a ed. São Paulo, Atheneu,1996: 792 p.

4. Enk I. Como reduzir o uso de Vancomicina em Unidades Neonatais, 2013. Available in: https://www.sbp.com. br/fileadmin/user_upload/2015/02/VANCOMICINADOCUMENTO-CIENTIFICO2013.pdf 2013. Accessed on February 19, 2020.

5. BRASIL. MINISTÉRIO DA SAÚDE. Atenção à Saúde do Recém-Nascido Guia para os Profissionais de Saúde Volume INTERVENÇÕES COMUNS, ICTERÍCIA E INFECÇÕES, 1ạ ed. Brasília, Editora MS, 2011: 164 p.

6. Guedes ALL, Cardoso AMJ, Cuconato GLB, et al. Diretrizes Clínicas, Protocolos Clínicos - Sepse Neonatal, 2013. Available in: www.fhemig.mg.gov.br. Accessed on February 19, 2020.

7. Luna CM, Rodríguez-Noriega E, Bavestrello L, et al. Treatment of methicillin-resistant Staphylococcus aureus in Latin America. Brazilian J Infect Dis. 2010;14:119-27.

8. Calil R, Caldas JPS. Uso Racional e Seguro de Antibióticos em Neonatologia, 2012. Available in:https://www.sbp.com. $\mathrm{br} /$ fileadmin/user_upload/pdfs/USO_RACIONAL_SEGURO_ ANTIBIOTICOS_NEONATOLOGIA2.pdf. Accessed on February 19, 2020.

9. Sutherland SM, Byrnes JJ, Kothari M, et al. AKI in hospitalized children: Comparing the pRIFLE, AKIN, and KDIGO definitions. Clin J Am Soc Nephrol, 2015,10(4):554-61.

10. Araujo MRE de. Hemocultura: recomendações de coleta, processamento e interpretação dos resultados. J Infect Control. 2012;1(1):8-19.

11. Organização Pan-Americana da Saúde. Centro Latino- 
Americano de Perinatologia, Saúde da Mulher e Reprodutiva. Prevenção de infecções relacionadas à assistência à saúde em neonatologia, 2016. Available in:https://iris.paho.org/ bitstream/handle/10665.2/34361/9789275719640-por. pdf?sequence=1\&isAllowed=y. Accessed on August 5, 2020.

12. Patel SJ, Saiman L. Antibiotic Resistance in Neonatal Intensive Care Unit Pathogens: Mechanisms, Clinical Impact, and Prevention Including Antibiotic Stewardship. Clin Perinatol. 2010;37(3):547-63.

13. Chiu CH, Michelow IC, Cronin J, et al. Effectiveness of a guideline to reduce vancomycin use in the neonatal intensive care unit. Pediatr Infect Dis J. 2011; 30(4):273-8.

14. Hemels MAC, Van Den Hoogen A, Verboon-Maciolek MA, et al. A seven-year survey of management of coagulasenegative staphylococcal sepsis in the neonatal intensive care unit: Vancomycin may not be necessary as empiric therapy. Neonatology,2011,100:180-5.

15. 1Sánchez PJ, Moallem M, Cantey JB, et al. Empiric therapy with vancomycin in the neonatal intensive care unit: let's "get smart" globally! J Pediatr. 2016; 92(5):432-5.

16. Stoll BJ, Hansen N, Fanaroff AA, et al. Late-Onset Sepsis in Very Low Birth Weight Neonates: The Experience of the NICHD Neonatal Research Network. Pediatrics, 2002,110(2):285-91.

17. Jean-Baptiste N, Benjamin DK, Cohen-Wolkowiez M, et al. Coagulase-Negative Staphylococcal Infections in the Neonatal Intensive Care Unit. Infect Control Hosp Epidemiol. 2011; 32(7):679-86

18. de Souza Rugolo LMS, Bentlin MR, Mussi-Pinhata M, et al. Late-Onset Sepsis in very Low Birth Weight Infants: A Brazilian Neonatal Research Network Study. J Trop Pediatr. 2014; 60(6):415-21.

19. Iosifidis E, Evdoridou I, Agakidou E, et al. Vancomycinresistant Enterococcus outbreak in a neonatal intensive care unit: epidemiology, molecular analysis and risk factors. Am J Infect Control. 2013; 41(10):857-861.

20. Rasigade JP, Raulin O, Picaud JC, et al. Methicillin-resistant Staphylococcus capitis with reduced vancomycin susceptibility causes late-onset sepsis in intensive care neonates. PLoS One. 2012;7(2):e31548.

21. Fahmey SS. Early-onset sepsis in a neonatal intensive care unit in beni suef, Egypt: Bacterial isolates and antibiotic resistance pattern. Korean J Pediatr. 2013;56(8):332-7.

22. Waheed M, Laeeq A, Maqbool S. The etiology of neonatal sepsis and patterns of antibiotic resistance. J Coll Physicians Surg Pak. 2003;13(8):449-52

23. Simonsen KA, Anderson-Berry AL, Delair SF, et al. Early-onset neonatal sepsis. Clin Microbiol Rev. 2014; 27(1):21-47.

24. Linder N, Lubin D, Hernandez A, et al. Duration of vancomycin treatment for coagulase-negative Staphylococcus sepsis in very low birth weight infants. Br J Clin Pharmacol. 2013;76(1):58-64.

25. Moffett BS, Morris J, Kam C, et al. Vancomycin associated acute kidney injury in pediatric patients. PLoS One. 2018,13(10).

26. Appel KL de A, Reis RBJ, Martins A de S, et al. Presença de Staphylococcus epidermidis em recém-nascidos com infecção grave em UTI neonatal. Perspect Exp e Clínicas, Inovações Biomédicas e Educ em Saúde. 2016;1:22-5. 